\title{
Validation of the new pathology staging system for progressive supranuclear palsy
}

\author{
Mayen Briggs ${ }^{1} \cdot$ Kieren Simon James Allinson $^{1} \cdot$ Maura Malpetti $^{2,3} \cdot$ Maria Grazia Spillantini $^{4}$. \\ James Benedict Rowe $\mathrm{e}^{1,2,3,5}$. Sanne Simone Kaalund ${ }^{2,3,4}$
}

Received: 17 January 2021 / Revised: 16 March 2021 / Accepted: 16 March 2021 / Published online: 28 March 2021

(c) The Author(s) 2021

Progressive supranuclear palsy (PSP) is a neurodegenerative disorder associated with neuroglial accumulation of 4-repeat tau protein [2]. Kovacs et al. [1] have recently proposed a new semi-quantitative six-stage system to categorise the severity of PSP pathology. Importantly, the system reduces reliance on regions with high risk of concomitant pathology and focusses on cell type-specific tau-pathology.

Here, we test the new PSP pathology staging system in an independent series of 35 PSP cases and test the potential association between pathology stage and clinical severity at death. We include tissue from 35 people with a clinical diagnosis of PSP (including $N=25$ with Richardson's syndrome and $N=10$ with other phenotypes; Movement Disorder Society 2017 criteria; Supplementary table 1, online resource). Donors had attended longitudinal clinical studies at the Cambridge Centre Parkinson-plus including assessment of clinical severity by the PSP rating scale (PSPRS) and cognitive performance by the revised Addenbrooke's Cognitive Examination (ACE-R). The left brain hemisphere was available for pathological evaluation (Supplementary methods, online resource) and following the guidelines from Kovacs et al. we rated regional tau cytopathology focussing

Sanne Simone Kaalund

ssk42@medschl.cam.ac.uk

1 Cambridge University Hospitals NHS Foundation Trust and the Cambridge Brain Bank, Cambridge CB2 2QQ, UK

2 Department of Clinical Neurosciences, University of Cambridge, Cambridge Biomedical Campus, Cambridge CB2 OSZ, UK

3 Cambridge Centre for Parkinson-Plus, University of Cambridge, Cambridge, UK

4 Department of Clinical Neurosciences, University of Cambridge, Cambridge Biomedical Campus, Clifford Allbutt Building, Hills Road, Cambridge CB2 0AH, UK

5 Medical Research Council Cognition and Brain Sciences Unit, University of Cambridge, Cambridge CB2 7EF, UK on astrocytic tau inclusions in striatum (STR), frontal and occipital cortices, and neuronal and oligodendroglia tau inclusions in globus pallidus (GP), subthalamic nucleus (STN), and cerebellum.

First, we selected ten random cases and, in each area, two authors independently rated tau pathology following the new staging system as described by Kovacs et al. [1]. The raters were in agreement in just 45/60 regions (75\%). Pallidum, cerebellum and occipital lobe had high agreement $(\geq 8 / 10)$, STN and frontal cortex intermediate (7/10), while STR had low agreement (4/10). This discrepancy was attributed to the lack of operational criteria and individual interpretations of the staging system ratings for each area, confounded by marked differences between regions in the absolute numbers of immunoreactive cells per field. We therefore formulated operational criteria with region-specific thresholds see Fig. 1c and Supplementary Fig. 1 (online resource) for a visual guide. With the new operational criteria the interrater agreement increased to $88 \%$ (52/59 regions), with high agreement for GP, STR, frontal cortex, occipital and cerebellum (9/10) and intermediate for STN (7/9). Integrating these operational criteria to the new staging system, $91 \%(32 / 35)$ of cases fitted readily into one of the six stages (Fig. 1a), including 9/10 of the cases with non-Richardson's phenotype (Fig. 1d and e).

We then tested whether the pathology staging was associated with demographic, age and clinical severity. There was no significant association between the pathological stages and age or symptom duration (Kruskal Wallis, $p>0.05$ ). The interval between death and last assessment of disease severity (PSPRS and ACE-R) varied from 24 days to 35 months. To account for the differences in interval between testing and death we took two approaches (1) weighting the analysis for this time interval (Clinical score $~$ PSP pathology stagelweight $=1$ /interval between assessment and death; Fig. 1b) and, (2) imputing the PSPRS and ACE-R scores at death from longitudinal assessments (imputed score $\sim$ PSP 


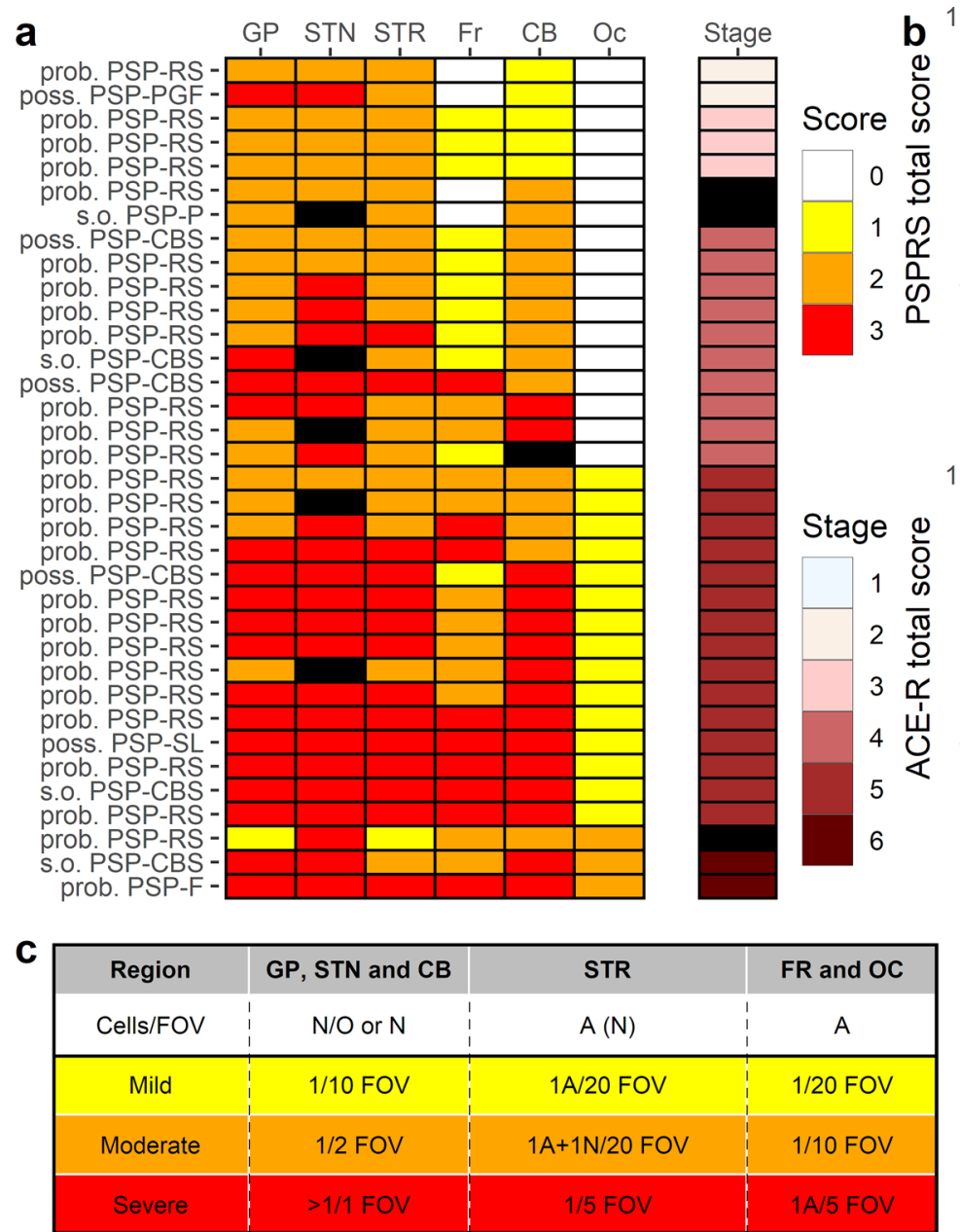

e poss. PSP-CBS

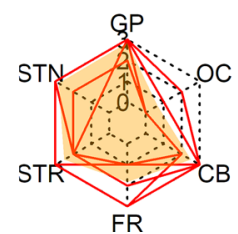

prob. PSP-F

poss. PSP-SL

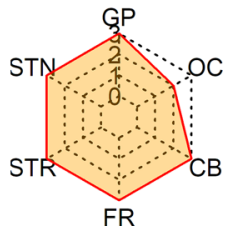

ANOVA $F(4,25)=3.0, p=0.04$
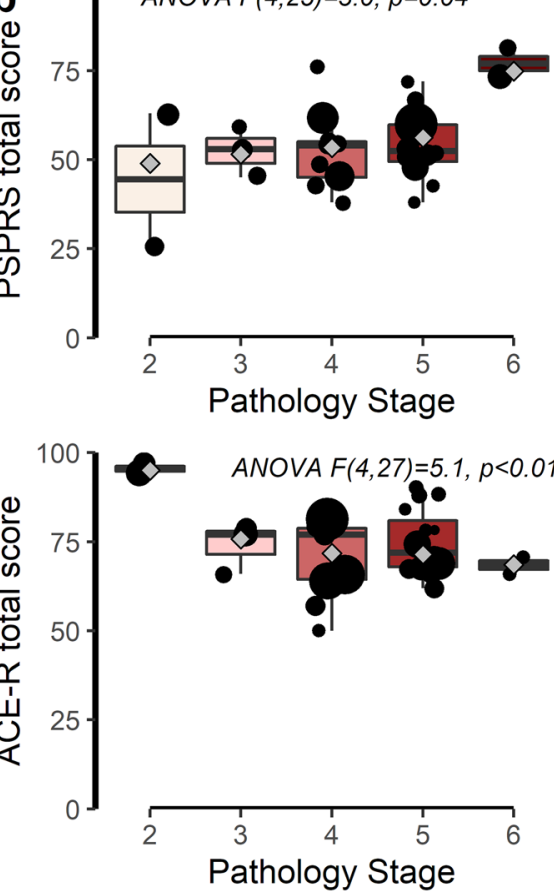

d

prob. PSP-RS

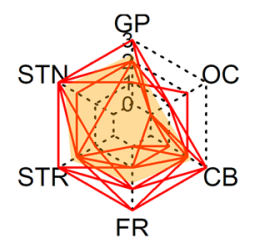

poss. PSP-PGF

$$
\text { s.o. PSP-P }
$$
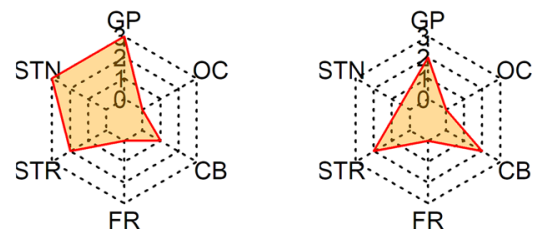

$\mathrm{FR}$
Fig. 1 Validation of pathology staging system for PSP. a Regional pathology scores (white to red) and pathology staging (light blue to brown). Black tiles indicate a missing region/no stage. Last diagnosis based on the movement disorder criteria is shown to the left of the tiles. b Pathology stage was correlated with clinical severity PSPRS and ACE-R. Individual data points are shown with black dots, boxplots show median, first and third quartiles, extending whiskers to $1.5 *$ interquartile range. Grey diamonds show the weighted group means, and the size of the points are proportional to the weight in the analysis. c Guide for tau pathology rating. d and e Cases with subcortical phenotypes, PSP-P and PSP-PGF, showed minimal pathology. Red lines show individual profiles of regional pathology and

pathology stage; Supplementary Fig. 2a, b and 3, 4, online resource). There was a significant association between pathology stage and PSPRS, both when weighting for time between last testing and death $(F(4,25)=30.0, p=0.036)$ orange fill the average group profiles. Striped lines show concentric hexagons representing no to severe (0-3) pathology. PSP progressive supranuclear palsy, prob. probable, poss. possible, s.o suggestive of, $R S$ Richardson's syndrome, $C B S$ corticobasal syndrome, $S L$ speech/ language variant, $F$ frontal, $P G F$ progressive gait freezing, $P$ parkinsonism, $G P$ globus pallidus, $O C$ occipital cortex, $F R$ frontal cortex, $C B$ cerebellum, STR striatum, STN subthalamic nucleus, PSPRS PSP Rating Scale, $A C E-R$ revised Addenbrooke's Cognitive Examination, $F O V$ field of view under $40 \times$ objective, $A$ tufted astrocytes, $N$ neuronal tangles and tau positive threads, $O$ coiled bodies, n.s. not significant, $p>0.05$

and using imputed PSPRS scores $(F(4,27)=2.8, p=0.045)$. There was a significant association between pathology stage and cognitive deficit, when weighting the analysis for time between testing and death $(F(4,27)=5.09, p=0.0035)$, but 
not using imputed ACE-R scores $(F(4,27)=2.43, p=0.07)$. Given the small group sizes for stages 2, 3 and 6, post hoc analysis were not performed.

Overall, our study supports the validity of the proposed PSP pathology staging system proposed by Kovacs et al. [1], being easy to implement in the day-to-day neuropathological evaluation (and retrospectively) as the regions required are routinely sampled for the pathological diagnosis of neurodegenerative disease. The proposed PSP staging schema is applicable across the spectrum of clinical PSP subtypes with $>90 \%$ of cases fulfilling staging criteria. In addition to the written description provided by Kovacs et al. we provide region-specific quantitative criteria along with a visual guide for the rating of tau pathology. Together with high compliance with the staging scheme, our findings suggest that the sequential distribution of tau pathology is associated with progressive clinical severity in PSP.

Supplementary Information The online version contains supplementary material available at https://doi.org/10.1007/s00401-021-02298-z.

Acknowledgements The authors would like to thank the research participants and caregivers, and the staff at the Cambridge Centre for Parkinson-Plus and the Department of Histopathology and Cytology at the Cambridge Brain Bank.

Author contribution All authors contributed to the study conception and design. Material preparation, data collection and analysis were performed by MB, KA, MM, and SSK. JR and MS critically revised and contributed important intellectual content to the manuscript. All authors read and approved the final manuscript.

Funding This work was funded by the Cambridge Brain Bank, Wellcome Trust (103838); Medical Research Council (146281), and the Cambridge Centre for Parkinson-plus (RG95450). The Cambridge Brain Bank is supported by the NIHR Cambridge Biomedical Research Centre. The views expressed are those of the authors and not necessarily those of the NIHR or the Department of Health and Social Care.

Data availability The datasets used are available from the corresponding author on reasonable request.

\section{Declarations}

Conflict of interests The authors declare that they have no conflict of interest.

Ethical approval The study ethics was approved by the Health Research Authority, NHS, England (IRAS- 202 802, "Neurodegeneration Research in Dementia"). The PiPPIN (Pick's Disease and Progressive Supranuclear Palsy: Prevalence and Incidence) Study was approved by Cambridge's research ethics committee. The study was conducted in accordance with the 1964 Helsinki declaration.

Open Access This article is licensed under a Creative Commons Attribution 4.0 International License, which permits use, sharing, adaptation, distribution and reproduction in any medium or format, as long as you give appropriate credit to the original author(s) and the source, provide a link to the Creative Commons licence, and indicate if changes were made. The images or other third party material in this article are included in the article's Creative Commons licence, unless indicated otherwise in a credit line to the material. If material is not included in the article's Creative Commons licence and your intended use is not permitted by statutory regulation or exceeds the permitted use, you will need to obtain permission directly from the copyright holder. To view a copy of this licence, visit http://creativecommons.org/licenses/by/4.0/.

\section{References}

1. Kovacs GG, Lukic MJ, Irwin DJ et al (2020) Distribution patterns of tau pathology in progressive supranuclear palsy. Acta Neuropathol 140:99-119. https://doi.org/10.1007/s00401-020-02158-2

2. Litvan I, Hauw JJ, Bartko JJ et al (1996) Validity and reliability of the preliminary NINDS neuropathologic criteria for progressive supranuclear palsy and related disorders. J Neuropathol Exp Neurol 55:97-105. https://doi.org/10.1097/00005072-19960 $1000-00010$

Publisher's Note Springer Nature remains neutral with regard to jurisdictional claims in published maps and institutional affiliations. 